\title{
Gap probabilities in the finite and scaled Cauchy random matrix ensembles
}

\author{
N.S. Witte, \\ Department of Mathematics and Statistics \\ \& School of Physics, University of Melbourne \\ Parkville, Victoria 3052, AUSTRALIA \\ and P.J. Forrester \\ Department of Mathematics and Statistics \\ University of Melbourne \\ Parkville, Victoria 3052, AUSTRALIA.
}

October 29, 2018

\begin{abstract}
The probabilities for gaps in the eigenvalue spectrum of finite $N \times N$ random unitary ensembles on the unit circle with a singular weight, and the related hermitian ensembles on the line with Cauchy weight, are found exactly. The finite cases for exclusion from single and double intervals are given in terms of second order second degree ODEs which are related to certain Painlevé-VI transcendents. The scaled cases in the thermodynamic limit are again second degree and second order, this time related to Painlevé-V transcendents. Using transformations relating the second degree ODE and transcendent we prove an identity for the scaled bulk limit which leads to a simple expression for the spacing p.d.f. We also relate all the variables appearing in the Fredholm determinant formalism to particular Painlevé transcendents, in a simple and transparent way, and exhibit their scaling behaviour.
\end{abstract}




\section{Introduction}

Our intention in this work is to study two intimately related ensembles of random matrices with unitary symmetry - the circular Jacobi unitary ensemble consisting of eigenvalues confined to the unit circle with a spectrum singularity, and the Cauchy ensemble on the real line. As we shall see, there is a mapping between these two ensembles which allows us to find all the results for the circular Jacobi ensemble from those of the Cauchy ensemble. The latter is a classical matrix ensemble on the real line, and as such there is a well established method for characterising the gap probabilities in terms of differential equations [13]. This avoids the clumsy and unnecessary work of recasting all of the formalism onto the unit circle. The circular Jacobi ensemble that we consider is one with an algebraic singularity in its spectrum, which in the log-gas picture of the eigenvalue p.d.f. can be interpreted as an impurity with variable charge fixed at $z=1$ interacting with the mobile unit charges representing the eigenvalues.

In general an ensemble of unitary matrices with unitary symmetry has an eigenvalue p.d.f. of the form

$$
\prod_{l=1}^{N} w_{2}\left(z_{l}\right) \prod_{1 \leq j<k \leq N}\left|z_{k}-z_{j}\right|^{2},
$$

$z=e^{i \theta}=e^{2 \pi i x / L}, \quad \theta \in[0,2 \pi), \quad x \in[0, L)$, while an ensemble of Hermitian matrices with unitary symmetry has an eigenvalue p.d.f. of the form

$$
\prod_{l=1}^{N} w_{2}\left(\lambda_{l}\right) \prod_{1 \leq j<k \leq N}\left|\lambda_{k}-\lambda_{j}\right|^{2} .
$$

The circular Jacobi ensemble with unitary symmetry, denoted as cJUE, is specified by the p.d.f. (1.1) with the weight function

$$
w_{2}(z)=|1-z|^{2 a} .
$$

In the case $a=1$ this is realised by the eigenvalues $z=e^{i \theta}$ from the ensemble of $(N+1) \times$ $(N+1)$ random unitary matrices, with all angles $\theta$ measured from any one eigenvalue. The Cauchy unitary ensemble, denoted by CyUE, is specified by (1.2) with

$$
w_{2}(\lambda)=\left(1+\lambda^{2}\right)^{-N-a}, \quad \lambda \in(-\infty, \infty) .
$$

It results from the cJUE by mapping the eigenvalues from the unit circle to the line tangent to its south-most point via a stereographic projection from its north-most point. Specifying points on the unit circle via an azimuthal angle $\theta$, so that $\theta=0$ corresponds to the north-most point and $\theta=-\pi, \pi$ to the south-most point, this is achieved by the mapping

$$
\lambda=\cot (\theta / 2),
$$

and we find

$$
\begin{aligned}
\prod_{i=1}^{N}\left|1-z_{i}\right|^{2 a} \prod_{1 \leq j<k \leq N}\left|z_{k}-z_{j}\right|^{2} d \theta_{1} \ldots d \theta_{N} & \\
& \propto \prod_{i=1}^{N} \frac{1}{\left(1+\lambda_{i}^{2}\right)^{N+a}} \prod_{1 \leq j<k \leq N}\left|\lambda_{k}-\lambda_{j}\right|^{2} d \lambda_{1} \ldots d \lambda_{N} .
\end{aligned}
$$


In (1.2), let us write

$$
w_{2}(\lambda)=e^{-2 V(\lambda)} \quad \text { and } \quad 2 V^{\prime}(\lambda)=\frac{g(\lambda)}{f(\lambda)},
$$

with $f(\lambda), g(\lambda)$ assumed polynomials in $\lambda$. In [1], the defining property of a classical ensemble in the random matrix context was identified as the operator

$$
\mathbf{n}=f \frac{d}{d x}+\left(\frac{f^{\prime}-g}{2}\right)
$$

increasing the degree of the polynomials by one. Thus for a classical ensemble

$$
\text { degree } f \leq 2, \quad \text { degree } g \leq 1 .
$$

Using this criterium one can check that the weight functions

$$
w_{2}(\lambda)=\left\{\begin{array}{l}
e^{-\lambda^{2}}, \quad \text { Hermite } \\
\lambda^{a} e^{-\lambda} \quad(\lambda>0), \quad \text { Laguerre } \\
(1-\lambda)^{\alpha}(1+\lambda)^{\beta} \quad(-1<\lambda<1), \quad \text { Jacobi }
\end{array}\right.
$$

are all classical. These weight functions all naturally occur as the eigenvalue p.d.f. for certain ensembles of Hermitian matrices based on matrices with independent Gaussian elements (see e.g. the Introduction section of [6]). Furthermore, for each of these cases the probability of a single eigenvalue free region which includes an endpoint of the support of the weight has been expressed in terms of the solution of a particular non-linear differential equation 13. The same has been done for the Hermite and symmetric Jacobi $(\alpha=\beta)$ ensembles when the eigenvalue free region consists of two disjoint intervals at either end of the support of the spectrum [14. The Cauchy weight function (1.4) is significant for the feature of satisfying the criterium (1.9) and thus being a fourth classical weight function. The orthogonal polynomial system defined by the Cauchy weight has been investigated in [12] and [2], where the polynomials have been found to be given by certain Jacobi polynomials of pure imaginary argument. Consequently we find some similarities with the Jacobi ensemble, but also sufficient differences to warrant separate attention. With $E_{2}\left(0 ; I ; w_{2}(\lambda) ; N\right)$ denoting the probability that there are no eigenvalues in the interval $I$ of an ensemble with eigenvalue p.d.f. (1.2), the probabilities to be calculated are

$$
E_{2}\left(0 ;(s, \infty) ;\left(1+\lambda^{2}\right)^{-N-a} ; N\right)
$$

and

$$
E_{2}\left(0 ;(-\infty,-s) \cup(s, \infty) ;\left(1+\lambda^{2}\right)^{-N-a} ; N\right)
$$

The quantity (1.11) gives the probability that there are no eigenvalues in the CyUE greater than $s$, and the quantity in (1.12) gives the probability that there are no eigenvalues in the CyUE with modulus greater than $s$. In terms of the circular Jacobi ensemble the probabilities corresponding to (1.11) and (1.12) are

$$
E_{2}\left(0 ;(0, x) ;|1-z|^{2 a} ; N\right)
$$

and

$$
E_{2}\left(0 ;(-x, x) ;|1-z|^{2 a} ; N\right)
$$


The details of the Cauchy ensemble are defined in Section 2, including the orthonormal polynomials and associated coefficients. In Section 3 the formalism of Tracy and Widom 13 giving coupled differential equations for the gap probability and some auxiliary quantities is revised. In Section 4 the coupled equations for the single interval are reduced to an ordinary differential equation specifying the probability (1.11), and this equation is shown to be related to a Painlevé-VI transcendent. This problem and the associated ODE are mapped back to the circular Jacobi ensemble and the thermodynamic limit taken of (1.13), and the resulting ODE and its transformation to a Painlevé-V type studied. A similar study is undertaken in Section 5 for the probabilities of exclusion from the double interval, namely (1.12) and the scaling limit of (1.14). 


\section{The Cauchy Ensemble and Orthogonal Polynomials}

Consider the problems of computing the probability that there are no eigenvalues in a region $I$ of the spectrum for an ensemble specified by (1.4). Suppose $I$ consists of $M$ disjoint intervals, so that with the endpoints of these intervals denoted by $\left\{a_{j}\right\}_{j=1}^{2 M}$,

$$
I=\bigcup_{m \geq 1}^{M}\left(a_{2 m-1}, a_{2 m}\right) .
$$

The probability of no eigenvalues being found in this interval is given by the general expression (see e.g. 甽)

$$
E(0 ; I)=1+\sum_{n=1}^{\infty} \frac{(-1)^{n}}{n !} \int_{I} d x_{1} \ldots \int_{I} d x_{n} \rho_{n}\left(x_{1}, \ldots, x_{n}\right)
$$

where $\rho_{n}$ is the $n$-point distribution function of the eigenvalue p.d.f. For Hermitian matrix ensembles with unitary symmetry the eigenvalue p.d.f. is proportional to (1.2), and the corresponding $n$-point distribution function is given in terms of the orthonormal polynomials $\left\{p_{j}(x)\right\}_{j=0,1,2, \ldots}$ associated with the weight function $w_{2}(x)$ according to the formula

$$
\rho_{n}\left(x_{1}, \ldots, x_{n}\right)=\operatorname{det}\left[K_{N}\left(x_{i}, x_{j}\right)\right]_{1 \leq i, j \leq n},
$$

where

$$
K_{N}(x, y)=\left[w_{2}(x) w_{2}(y)\right]^{1 / 2} \sum_{l=0}^{N-1} p_{l}(x) p_{l}(y) .
$$

Substituting into (2.2), the Fredholm theory of integral operators then gives

$$
E(0 ; I)=\operatorname{det}\left(\mathbb{I}-\mathbb{K}_{N}\right),
$$

where $\mathbb{K}_{N}$ is the integral operator with kernel $K_{N}(x, y)$ defined on the interval $I$. A crucial point is that (2.4) can be summed according to the Christoffel-Darboux formula and so written in the special form [9]

$$
K_{N}(x, y)=\frac{\phi(x) \psi(y)-\phi(y) \psi(x)}{x-y},
$$

where with $a_{N}$ denoting the coefficient of $x^{N}$ in $p_{N}(x)$

$$
\begin{aligned}
& \phi(x)=\left(\frac{a_{N-1}}{a_{N}} w_{2}(x)\right)^{1 / 2} p_{N}(x), \\
& \psi(x)=\left(\frac{a_{N-1}}{a_{N}} w_{2}(x)\right)^{1 / 2} p_{N-1}(x) .
\end{aligned}
$$

Now, the Cauchy weight is defined by (1.4) where $N \in \mathbb{Z}^{+}$and $a \in \mathbb{R}^{+}$. This weight defines an orthogonal polynomial system only up to the degree $n=N$ and not for higher degrees, however this is of no consequence as we only require those below $N$. The Cauchy weight is closely related to the symmetric Jacobi weight $\left(1-\lambda^{2}\right)^{\alpha}$, as can be seen from the following integration identity [幽] 
Lemma 1 For analytic functions $f(x)$, such that the following integrals exist and $\Re(\alpha)>-1$,

$$
\int_{-\infty}^{+\infty} d x f(i x)\left(1+x^{2}\right)^{\alpha}=\tan (\pi \alpha) \int_{-1}^{1} d x f(x)\left(1-x^{2}\right)^{\alpha}
$$

Using (2.8), the fact that

$$
\int_{-1}^{1} d x\left(1-x^{2}\right)^{\alpha} P_{m}^{(\alpha, \alpha)}(x) P_{n}^{(\alpha, \alpha)}(x)=\frac{2^{2 \alpha+1} \Gamma^{2}(\alpha+n+1)}{(2 \alpha+2 n+1) \Gamma(n+1) \Gamma(2 \alpha+n+1)} \delta_{m, n},
$$

with $P_{n}^{(\alpha, \beta)}(x)$ denoting the usual Jacobi polynomial, allows us to conclude that

$$
p_{n}^{\mathrm{Cy}}(x)=i^{n} 2^{N+a}\left[\frac{n !(N+a-n-1 / 2) \Gamma^{2}(N+a-n)}{2 \pi \Gamma(2 N+2 a-n)}\right]^{1 / 2} P_{n}^{(-N-a,-N-a)}(i x),
$$

are the orthonormal polynomials for the Cauchy weight. From the definition of the $P_{n}^{(\alpha, \beta)}(x)$ it follows from (2.10) that the coefficient of $x^{n}$ required in (2.7) is such that

$$
\frac{a_{n-1}^{\mathrm{Cy}}}{a_{n}^{\mathrm{Cy}}}=1 / 2\left[\frac{n(2 N+2 a-n)}{(N+a-n+1 / 2)(N+a-n-1 / 2)}\right]^{1 / 2} .
$$

A common feature of the classical orthogonal polynomials is that $\phi(x)$ and $\psi(x)$ satisfy the recurrence-differential relations

$$
\begin{aligned}
& m(x) \phi^{\prime}(x)=A(x) \phi(x)+B(x) \psi(x), \\
& m(x) \psi^{\prime}(x)=-C(x) \phi(x)-A(x) \psi(x),
\end{aligned}
$$

where the coefficient functions $m(x), A(x), B(x), C(x)$ are polynomials in $x$. Making use of the differentiation formula

$$
\begin{aligned}
(2 n+\alpha+\beta)\left(1-x^{2}\right) \frac{d}{d x} P_{n}^{(\alpha, \beta)}(x)=n[\alpha-\beta-(2 n+\alpha+\beta) x] & P_{n}^{(\alpha, \beta)}(x) \\
& +2(n+\alpha)(n+\beta) P_{n-1}^{(\alpha, \beta)}(x),
\end{aligned}
$$

and the three term recurrence for the Jacobi polynomials gives that 2.12 holds for the Cauchy ensemble with [4]

$$
\begin{aligned}
& m(x)=1+x^{2}, \\
& A(x)=-a x, \\
& B(x)=\left[N(N+2 a) \frac{2 a-1}{2 a+1}\right]^{1 / 2}=\beta_{0}, \\
& C(x)=\left[N(N+2 a) \frac{2 a+1}{2 a-1}\right]^{1 / 2}=\gamma_{0},
\end{aligned}
$$

with $n=N$.

Tracy and Widom [13] have developed a formalism based on the integral operator determinant formula (2.5), and which makes essential use of the special structures (2.6) and (2.12), to derive a set of coupled differential equations for $E_{2}(0 ; I)$ and some auxiliary quantities. The salient features of this theory will be revised in the next section. 


\section{The General Formalism}

Our goal is to characterise the probabilities (1.11) and (1.12) as the solution of certain nonlinear differential equations. Following [13] this is achieved by specifying partial differential equations for the quantities $q_{j}, p_{j}, u, v, w$ and $R\left(a_{j}, a_{k}\right)$ defined henceforth.

Definition 1 Let $A \doteq A(x, y)$ denote that the integral operator $A$ has kernel $A(x, y)$. Then the kernels $\rho(x, y)$ and $R(x, y)$ are specified by

$$
\begin{aligned}
(1-K)^{-1} & \doteq \rho(x, y), \\
K(1-K)^{-1} & \doteq R(x, y),
\end{aligned}
$$

The operator $K(1-K)^{-1}$ is called the resolvent and $R(x, y)$ the resolvent kernel.

Definition 2 For $k \in \mathbb{Z}_{\geq 0}$ the functions $Q_{k}$ and $P_{k}$ are defined by

$$
\begin{aligned}
Q_{k}(x) & =\int_{I} d y \rho(x, y) y^{k} \phi(y), \\
P_{k}(x) & =\int_{I} d y \rho(x, y) y^{k} \psi(y),
\end{aligned}
$$

and their values at the endpoints $a_{j}$ of $I$ are denoted $q_{k j}, p_{k j}$ so that

$$
\begin{aligned}
& q_{k j}=Q_{k}\left(a_{j}\right) \equiv \lim _{x \rightarrow a_{j}} Q_{k}(x), \\
& p_{k j}=P_{k}\left(a_{j}\right) \equiv \lim _{x \rightarrow a_{j}} P_{k}(x) .
\end{aligned}
$$

Where there is no confusion, we denote $q_{0 j}, p_{0 j}$ by $q_{j}, p_{j}$.

Definition 3 The inner products $u, v, w$ are defined by

$$
\begin{aligned}
& u=\langle\phi \mid Q\rangle=\int_{I} d y Q_{0}(y) \phi(y) \\
& v=\langle\psi \mid Q\rangle=\int_{I} d y Q_{0}(y) \psi(y)=\langle\phi \mid P\rangle=\int_{I} d y P_{0}(y) \phi(y) \\
& w=\langle\psi \mid P\rangle=\int_{I} d y P_{0}(y) \psi(y) .
\end{aligned}
$$

The coupled differential equations come in two types. There is a set of universal equations which are independent of the recurrence-differential equations (2.12), and a set of equations which depend on the details of (2.12). Let us first present the former.

Proposition 1 For general functions $\phi(x), \psi(x)$ we have the relations

$$
\frac{\partial}{\partial a_{j}} \log \operatorname{det}(1-K)=(-1)^{j-1} R\left(a_{j}, a_{j}\right)
$$

and for $j \neq k$,

$$
R\left(a_{j}, a_{k}\right)=\frac{q_{j} p_{k}-q_{k} p_{j}}{a_{j}-a_{k}}
$$


and

$$
\frac{\partial}{\partial a_{k}} R\left(a_{j}, a_{j}\right)=(-1)^{k} R\left(a_{j}, a_{k}\right) R\left(a_{k}, a_{j}\right)
$$

along with

$$
\begin{aligned}
& \frac{\partial q_{j}}{\partial a_{k}}=(-1)^{k} R\left(a_{j}, a_{k}\right) q_{k}, \\
& \frac{\partial p_{j}}{\partial a_{k}}=(-1)^{k} R\left(a_{j}, a_{k}\right) p_{k},
\end{aligned}
$$

for $j \neq k$ and

$$
\begin{aligned}
\frac{\partial u}{\partial a_{k}} & =(-1)^{k} q_{k}^{2}, \\
\frac{\partial v}{\partial a_{k}} & =(-1)^{k} q_{k} p_{k}, \\
\frac{\partial w}{\partial a_{k}} & =(-1)^{k} p_{k}^{2} .
\end{aligned}
$$

The second set of equations, which depend on the details of (2.12), give the $j=k$ cases of (3.6) and (3.8). Now for the Cauchy weight (1.4) we know from (2.14) the equations (2.12) hold for $m(x)$ a quadratic and $A(x), B(x), C(x)$ linear functions, and thus of the general form

$$
\begin{aligned}
& m(x)=\mu_{0}+\mu_{1} x+\mu_{2} x^{2}, \\
& A(x)=\alpha_{0}+\alpha_{1} x, \\
& B(x)=\beta_{0}+\beta_{1} x, \\
& C(x)=\gamma_{0}+\gamma_{1} x .
\end{aligned}
$$

One then has the following equations [13].

Proposition 2 In the case that $\phi(x), \psi(x)$ satisfy the equations (2.19) with coefficient functions (3.10) we have

$$
\begin{gathered}
m_{i} \frac{\partial q_{i}}{\partial a_{i}}=\left[\alpha_{0}+\alpha_{1} a_{i}+\gamma_{1} u-\beta_{1} w-\mu_{2} v\right] q_{i} \\
+\left[\beta_{0}+\beta_{1} a_{i}+2 \alpha_{1} u+2 \beta_{1} v+\mu_{2} u\right] p_{i} \\
-\sum_{k \neq i}^{2 M}(-1)^{k} R\left(a_{i}, a_{k}\right) q_{k} m_{k}, \\
m_{i} \frac{\partial p_{i}}{\partial a_{i}}=\left[-\gamma_{0}-\gamma_{1} a_{i}+2 \gamma_{1} v+2 \alpha_{1} w-\mu_{2} w\right] q_{i} \\
+\left[-\alpha_{0}-\alpha_{1} a_{i}+\beta_{1} w-\gamma_{1} u+\mu_{2} v\right] p_{i} \\
-\sum_{k \neq i}^{2 M}(-1)^{k} R\left(a_{i}, a_{k}\right) p_{k} m_{k},
\end{gathered}
$$


and

$$
\begin{gathered}
m_{i} R\left(a_{i}, a_{i}\right)=\left[\gamma_{0}+\gamma_{1} a_{i}-2 \gamma_{1} v-2 \alpha_{1} w+\mu_{2} w\right] q_{i}^{2} \\
+\left[\beta_{0}+\beta_{1} a_{i}+2 \alpha_{1} u+2 \beta_{1} v+\mu_{2} u\right] p_{i}^{2} \\
+\left[\alpha_{0}+\alpha_{1} a_{i}+\gamma_{1} u-\beta_{1} w-\mu_{2} v\right] 2 q_{i} p_{i} \\
+\sum_{k \neq i}^{2 M}(-1)^{k} m_{k} \frac{\left[q_{i} p_{k}-p_{i} q_{k}\right]^{2}}{a_{i}-a_{k}}
\end{gathered}
$$

and furthermore,

$$
\begin{aligned}
\frac{\partial}{\partial a_{i}}\left[m_{i} R\left(a_{i}, a_{i}\right)\right]=2 \alpha_{1} q_{i} p_{i} & +\beta_{1} p_{i}^{2}+\gamma_{1} q_{i}^{2} \\
& -\sum_{k \neq i}^{2 M}(-1)^{k} m_{k} R^{2}\left(a_{i}, a_{k}\right),
\end{aligned}
$$

where $m_{i}=m\left(a_{i}\right)$. 


\section{The Single Interval}

We first consider the probability for the interval $(s, \infty)$ to be free of eigenvalues so that $a_{1}=s$ and $a_{2}=\infty$. We shall adopt the conventions $q_{1}, p_{1}=q, p$ and $R=R(s, s)$, noting that $q_{2}, p_{2}=0$.

Proposition 3 The coupled differential equations for the finite $N$ CyUE on the interval $(s, \infty)$ for general $\alpha, \beta$ are

$$
\begin{aligned}
{\left[\ln E_{2}\right]^{\prime} } & =R \\
u^{\prime} & =-q^{2} \\
v^{\prime} & =-q p \\
w^{\prime} & =-p^{2}, \\
\left(1+s^{2}\right) q^{\prime} & =-[a s+v] q+\left[\beta_{0}-u(2 a-1)\right] p, \\
\left(1+s^{2}\right) p^{\prime} & =-\left[\gamma_{0}+w(2 a+1)\right] q+[a s+v] p, \\
\left(1+s^{2}\right) R & =\left[\gamma_{0}+w(2 a+1)\right] q^{2}+\left[\beta_{0}-u(2 a-1)\right] p^{2}-[a s+v] 2 q p \\
{\left[\left(1+s^{2}\right) R\right]^{\prime} } & =-2 a q p,
\end{aligned}
$$

Proof (sketch) - The first equation follows from (3.5) and (2.5). The next three (4.2)-(4.4) follow from (3.9) and the pair (4.5), (4.6) follow from (3.11). Equation (4.7) follows from (3.12), while (4.8) follows from (3.13).

The boundary conditions satisfied by $R(s, s)$ as $s \rightarrow \infty$, deduced from the fact that in this limit $R(s, s) \sim K_{N}(s, s)$, can be expressed as

$$
\begin{aligned}
R(s, s) \sim(-1)^{N-1} & \frac{2^{2(N+a)}}{4 \pi} \frac{N ! \Gamma^{2}(a)}{\Gamma(N+2 a)}\left(1+s^{2}\right)^{-N-a-1} \\
\times & \left\{-N(N+2 a)\left[P_{N}^{(-N-a,-N-a)}(i s)\right]^{2}\right. \\
& -2 i a^{2} s P_{N}^{(-N-a,-N-a)}(i s) P_{N-1}^{(-N-a,-N-a)}(i s) \\
& \left.+a^{2}\left[P_{N-1}^{(-N-a,-N-a)}(i s)\right]^{2}\right\}
\end{aligned}
$$

or considering only the first two leading order terms

$$
\begin{aligned}
\left(1+s^{2}\right) R(s, s) \sim \frac{2^{2 a}}{\pi} \frac{\Gamma(1+N+2 a) \Gamma^{2}(a+1)}{\Gamma(N) \Gamma^{2}(2 a+2)} s^{-2 a} \\
\times\left[2 a+1-s^{-2} \frac{a}{2 a+3}\left(2 N^{2}+4 a N+4 a^{2}+4 a+1\right)\right] .
\end{aligned}
$$

We now indicate how to reduce such a system to a single second order differential equation for $R=R(s)=R(s, s)$. 
Proposition 4 The coupled set of ODEs given in Proposition 3 reduce to the second order ODE for $\sigma(s)=\left(1+s^{2}\right) R(s)$,

$$
\begin{gathered}
\left(1+s^{2}\right)^{2}\left(\sigma^{\prime \prime}\right)^{2}+4\left(1+s^{2}\right)\left(\sigma^{\prime}\right)^{3}-8 s \sigma\left(\sigma^{\prime}\right)^{2}+4 \sigma^{2}\left(\sigma^{\prime}-a^{2}\right) \\
+8 a^{2} s \sigma \sigma^{\prime}+4\left[N(N+2 a)-a^{2} s^{2}\right]\left(\sigma^{\prime}\right)^{2}=0 .
\end{gathered}
$$

Proof - We note from (4.3) and (4.8) the integral

$$
\left(1+s^{2}\right) R=2 a v,
$$

where the constant of integration has been set to zero because $v,\left(1+s^{2}\right) R \rightarrow 0$ as $s \rightarrow \infty$. Now we show how a second integral of the motion can be found. Utilising this first integral and (4.7) we have

$$
\left[\gamma_{0}+w(2 a+1)\right] q^{2}+\left[\beta_{0}-u(2 a-1)\right] p^{2}-[a s+v] 2 q p-2 a v=0,
$$

while adding $p$ times (4.5) and $q$ times (4.6) yields another equation quadratic in the $p$ and $q$

$$
\left(1+s^{2}\right)(p q)^{\prime}+\left[\gamma_{0}+w(2 a+1)\right] q^{2}-\left[\beta_{0}-u(2 a-1)\right] p^{2}=0 .
$$

Now the idea is to combine these two equations in such a way so that use of the relations (4.24.4) to express products of $q, p$ in terms of derivatives of $u, v, w$ leads to an exact derivative. Such a combination is (4.13) minus $2 a$ times (4.14), which when equations (4.2 4.4 ) are employed yields

$$
\begin{aligned}
& 2 a\left(1+s^{2}\right) v^{\prime \prime}+2 a s v^{\prime}+2 v v^{\prime}-2 a v \\
& +(2 a-1) u^{\prime}\left[\gamma_{0}+w(2 a+1)\right]-(2 a+1) w^{\prime}\left[\beta_{0}-u(2 a-1)\right]=0 .
\end{aligned}
$$

This can be rewritten as

$$
\begin{aligned}
& 2 a\left(1+s^{2}\right) v^{\prime \prime}+4 a s v^{\prime} \\
& +2 v v^{\prime}-2 a v-2 a s v^{\prime} \\
& -\left[\beta_{0}-u(2 a-1)\right]^{\prime}\left[\gamma_{0}+w(2 a+1)\right]-\left[\gamma_{0}+w(2 a+1)\right]^{\prime}\left[\beta_{0}-u(2 a-1)\right]=0,
\end{aligned}
$$

which is a perfect derivative. Given that the boundary conditions as $s \rightarrow \infty$ imply $u, v, w \rightarrow 0$, the integration constant can be determined, leading to the second integral

$$
\left[\beta_{0}-u(2 a-1)\right]\left[\gamma_{0}+w(2 a+1)\right]=\beta_{0} \gamma_{0}+\left(1+s^{2}\right) \sigma^{\prime}-s \sigma+\frac{1}{4 a^{2}} \sigma^{2} .
$$

We seek now to utilise all these equations to arrive at a second order ODE for $\sigma(s)$, and to do so we note the pair of equations

$$
-\frac{1}{2 a}\left(1+s^{2}\right) \sigma^{\prime \prime}=\left[\beta_{0}-u(2 a-1)\right] p^{2}-\left[\gamma_{0}+w(2 a+1)\right] q^{2},
$$

which is a consequence of (4.14) and (4.12), and

$$
\sigma-\frac{1}{2 a^{2}}\left(\sigma+2 a^{2} s\right) \sigma^{\prime}=\left[\beta_{0}-u(2 a-1)\right] p^{2}+\left[\gamma_{0}+w(2 a+1)\right] q^{2} .
$$

We seek to eliminate the two terms appearing on the right-hand sides of these last equations by forming the difference of their squares and using their cross product from the second integral (4.17). The resulting equation is the ODE stated in the proposition. 


\subsection{Reduction to Painlevé Type}

It is immediately clear that we can make an identification of our ODE with the master Painlevé equation for second order second degree ordinary differential equations with Painlevé integrability of Cosgrove and Scoufis, [3] equation (5.1), where in terms of this equation we have $g(s)=1+s^{2}, h(s)=N(N+2 a)-a^{2} s^{2}, f(s)=0$, and the coefficients $c_{1}=0, c_{2}=$ $1, c_{3}=0, c_{4}=1, c_{5}=-a^{2}, c_{6}=0, c_{7}=N(N+2 a), c_{8}=0, c_{9}=0, c_{10}=0$.

Proposition 5 The function $\sigma(s)=\left(1+s^{2}\right) R(s)$ for the Cauchy Unitary Ensemble on the interval $(s, \infty)$ is related to a solution of the Painlevé VI transcendent $\omega(t)$ with the parameters

$$
\alpha=1 / 2, \quad \beta=-1 / 2(N+a)^{2}, \quad \gamma=1 / 2(N+a)^{2}, \quad \delta=1 / 2-2 a^{2},
$$

by the gauge

$$
\begin{aligned}
t & =1 / 2(1-i s), \\
\eta(t) & =\frac{1}{2 i}\left(\sigma(s)-a^{2} s\right),
\end{aligned}
$$

and Bäcklund transformations

$$
\begin{aligned}
& \eta=\frac{t^{2}(t-1)^{2}}{4 \omega(\omega-1)(\omega-t)}\left\{\dot{\omega}-\frac{\omega(\omega-1)}{t(t-1)}\right\}^{2}-\frac{1}{4}(N+a)^{2} \frac{\omega-t}{\omega(\omega-1)}+1 / 2 a^{2}\left(1-2 t \frac{\omega-1}{\omega-t}\right), \\
& \dot{\eta}=-\frac{t(t-1)}{4 \omega(\omega-1)}\left\{\dot{\omega}-\frac{\omega(\omega-1)}{t(t-1)}\right\}^{2}+\frac{1}{4}(N+a)^{2} \frac{(\omega-t)^{2}}{t(t-1) \omega(\omega-1)} .
\end{aligned}
$$

All that is required to make an explicit relation to one of the Painlevé transcendents is to find the right gauge transformation to put (4.11) into the canonical form of (SD-Ia) in [3]. Given that our function $g(s)$ has zeros at $s_{1}=\infty, s_{2}=i, s_{3}=-i$ this determines $a_{1}, a_{2}, a_{3}, a_{4}$ in the general gauge transformation

$$
t=\frac{a_{1} s+a_{2}}{a_{3} s+a_{4}} \quad \eta=\frac{a_{5} \sigma+a_{6} s+a_{7}}{a_{3} s+a_{4}},
$$

leaving $a_{5}, a_{6}, a_{7}$. The coefficient $a_{5}$ is found from matching the cubic terms in the ODE (all three cubic terms must be matched with the single coefficient), whilst $a_{6}, a_{7}$ are determined by the $\eta^{2}, \eta \dot{\eta}$ terms. This determines the gauge transformation as given in (4.21). The free parameters in the canonical form are then found to be

$$
\begin{aligned}
& A_{1}=3 a^{2}+N(N+2 a), \\
& A_{2}=0, \\
& A_{3}=3 a^{4}+2 a^{2} N(N+2 a), \\
& A_{4}=a^{6}+a^{4} N(N+2 a) .
\end{aligned}
$$

From these four coefficients one solution for the Painlevé-VI transcendent coefficients is given in (4.20) and the corresponding Bäcklund transformation by (4.22). Other solution sets are possible, which are manifestations of Schlesinger transformations, and all are tabulated below in Table 1. 


\begin{tabular}{|c|c|c|c|}
\hline$\alpha=$ & $\beta=$ & $\gamma=$ & $\delta=$ \\
\hline $1 / 2$ & $-1 / 2(N+a)^{2}$ & $1 / 2(N+a)^{2}$ & $1 / 2-2 a^{2}$ \\
\hline $1 / 2(1 \pm 2 a)^{2}$ & $-1 / 2(N+a)^{2}$ & $1 / 2(N+a)^{2}$ & $1 / 2$ \\
\hline $1 / 2[1 \pm(N+2 a)]^{2}$ & $-1 / 2 a^{2}$ & $1 / 2 a^{2}$ & $1 / 2\left(1-N^{2}\right)$ \\
\hline $1 / 2[1 \pm N]^{2}$ & $-1 / 2 a^{2}$ & $1 / 2 a^{2}$ & $1 / 2\left[1-(N+2 a)^{2}\right]$ \\
\hline $1 / 2[1 \pm(N+a)]^{2}$ & 0 & $2 a^{2}$ & $1 / 2\left[1-(N+a)^{2}\right]$ \\
\hline $1 / 2[1 \pm(N+a)]^{2}$ & $-2 a^{2}$ & 0 & $1 / 2\left[1-(N+a)^{2}\right]$ \\
\hline $1 / 2[1 \pm a]^{2}$ & $-1 / 2 N(N+2 a)$ & $2 a^{2}+1 / 2 N(N+2 a)$ & $1 / 2\left(1-a^{2}\right)$ \\
\hline $1 / 2[1 \pm a]^{2}$ & $2 a^{2}-1 / 2 N(N+2 a)$ & $1 / 2 N(N+2 a)$ & $1 / 2\left(1-a^{2}\right)$ \\
\hline
\end{tabular}

Table 1: Painléve parameters for the P-VI transcendents characterising the gap p.d.f. for the Cauchy Hermitian random matrix ensemble on the interval $(s, \infty)$.

\subsection{Special Cases of low $N$}

In this part we present the calculations for the first two finite- $N$ cases, that is $N=1$ and $N=2$, by direct means using the probability $E_{2}(0 ; I)$.

Proposition 6 The probability (1.11) for $N=1$ and the associated quantities are given in terms of the Gauss hypergeometric function ${ }_{2} F_{1}(a, b ; c ; z)$ by

$$
\begin{aligned}
E_{2}(0 ; I) & =X, \\
\sigma(s) & =\frac{\Gamma(a+1)}{\sqrt{\pi} \Gamma(a+1 / 2)} \frac{\left(1+s^{2}\right)^{-a}}{E_{2}(0 ; I)},
\end{aligned}
$$

and similarly for the probability with $N=2$,

$$
\begin{aligned}
E_{2}(0 ; I) & =X^{2}-\frac{a \Gamma(a+1)}{\sqrt{\pi} \Gamma(a+3 / 2)} s\left(1+s^{2}\right)^{-a-1} X-\frac{\Gamma^{2}(a+1)}{2 \pi \Gamma(a+1 / 2) \Gamma(a+3 / 2)}\left(1+s^{2}\right)^{-2 a-1}, \\
\sigma(s) & =\frac{\Gamma(a+2)}{\sqrt{\pi} \Gamma(a+3 / 2)} \frac{\left(1+s^{2}\right)^{-a-1}}{E_{2}(0 ; I)}\left\{\left[1+(2 a+1) s^{2}\right] X+\frac{\Gamma(a+1)}{\sqrt{\pi} \Gamma(a+1 / 2)} s\left(1+s^{2}\right)^{-a}\right\}
\end{aligned}
$$

where

$$
\begin{aligned}
X & =1-\frac{\Gamma(a+1)}{2 \sqrt{\pi} \Gamma(a+3 / 2)} s^{-2 a-1}{ }_{2} F_{1}\left(a+1, a+1 / 2 ; a+3 / 2 ;-s^{-2}\right), \\
& =1 / 2+\frac{\Gamma(a+1)}{\sqrt{\pi} \Gamma(a+1 / 2)} s_{2} F_{1}\left(a+1,1 / 2 ; 3 / 2 ;-s^{2}\right),
\end{aligned}
$$

Proof - These follow from the integral representations and transformation formulae of the Gauss hypergeometric functions.

One can show that these two specific cases are solutions of the second order differential equation (4.11) and satisfy the boundary conditions (4.10), after noting the differentiation formula for the Gauss hypergeometric function. 


\subsection{The Thermodynamic Limit for the Circular Jacobi Ensemble}

Using the stereographic projection we map the extended real line back onto the circle of circumference $L$ via

$$
s=\cot \frac{\pi x}{L} \quad x \in[0, L) .
$$

Now as

$$
E_{2}\left(0 ;(s, \infty) ;\left(1+s^{2}\right)^{-N-a} ; N\right)=\exp \left(-\int_{s}^{\infty} d t \frac{\sigma(t)}{1+t^{2}}\right)
$$

this gap probability is then expressed as

$$
E_{2}\left(0 ;(0, x) ;\left|1-e^{2 \pi i y / L}\right|^{2 a} ; N\right)=\exp \left(-\frac{\pi}{L} \int_{0}^{x} d y \sigma(\cot \pi y / L)\right) .
$$

One is then interested in taking the scaling limit, interpreted as the thermodynamic limit, in which both $N, L \rightarrow \infty$ with a fixed density $\rho=N / L$. Evidently the natural quantity to consider in this limit is

$$
\frac{1}{N} \sigma(\cot \pi x / L) \mapsto \sigma(\pi \rho x),
$$

and so we investigate the above limit of (4.11) in terms of this new variable.

Proposition 7 In the thermodynamic limit the scaled variable $\tau_{a}(x) \equiv x \sigma(x)$ satisfies the second order second degree ordinary differential equation

$$
x^{2}\left(\ddot{\tau}_{a}\right)^{2}-4\left(x \dot{\tau}_{a}-\tau_{a}\right) \dot{\tau}_{a}^{2}-4 a^{2} \dot{\tau}_{a}^{2}+4\left(x \dot{\tau}_{a}-\tau_{a}\right)^{2}=0,
$$

subject to the boundary conditions

$$
\tau_{a}(x) \underset{x \rightarrow 0}{\sim} \frac{(1 / 2 x)^{2 a+1}}{\Gamma(a+1 / 2) \Gamma(a+3 / 2)} .
$$

Proof - This follows directly from the definition (4.31) under the map (4.28) of (4.11).

Proposition 8 The scaled variable $\tau_{a}(x)$ is determined by a solution of the Painlevé- $V$ transcendent $u(x)$ with parameters

$$
\alpha=1 / 2(1 \mp 2 a)^{2}, \quad \beta=0, \quad \gamma=0, \quad \delta=2,
$$

and is related by

$$
\begin{aligned}
& -\tau_{a}=\frac{1}{4 u}\left[\frac{x u^{\prime}}{u-1}-u\right]^{2}-a^{2} u+\frac{x^{2} u}{(u-1)^{2}}, \\
& -\dot{\tau}_{a}=-\frac{x}{4 u(u-1)}\left[u^{\prime}-(1 \mp 2 a) \frac{u(u-1)}{x}\right]^{2}-\frac{x u}{u-1} .
\end{aligned}
$$


Proof - The equation (4.32) falls into the class SD-I.b, equation (5.5) of [3] under $\tau_{a} \mapsto-\tau_{a}$ and so it is a matter of finding the appropriate gauge transformation. Clearly the parameters $a_{2}=a_{3}=0, a_{1}=a_{4}=a_{5}=1$, and this leaves $a_{6}, a_{7}$. Under such a mapping the canonical equation can be compared with our (4.32), and thus we find $a_{6}=0, a_{7}=-a^{2}$ and $A_{1}=$ $-4, A_{2}=-8 a^{2}, A_{3}=0, A_{4}=-4 a^{4}$. Solving for the Painlevé-V transcendent parameters and the transformation then leads to the results in the above proposition. Another valid solution has the parameters $\alpha=1 / 2, \beta=-2 a^{2}, \gamma=0, \delta=2$.

In the case $a=0$ the eigenvalue p.d.f. defining the cJUE is identical to the eigenvalue p.d.f. for the CUE of random unitary matrices. It is well known that in the scaled thermodynamic limit the $n$-point distribution function then has the structure (2.3) with $K_{N}(x, y)$ replaced by $K(x, y)$, which in turn has the explicit form

$$
K(x, y)=\frac{\sin \pi \rho(x-y)}{\pi(x-y)}
$$

For this scaled ensemble it is a celebrated result of Jimbo et al 10 that

$$
E_{2}(0 ;(0, x))=\exp \left(-\int_{0}^{\pi \rho x} \frac{\tau(y)}{y} d y\right)
$$

where $\tau(x)$ satisfies the equation (4.32) with $a=0$.

We have already remarked in the Introduction that the case $a=1$ of the cJUE is also related to the CUE, by measuring angles from a particular eigenvalue, taken as the origin, in the latter. Thus

$$
\left.E_{2}\left(0 ;(0, \theta) ; w_{2}(\theta)=1 ; N+1\right)\right|_{\text {given an eigenvalue at } \theta=0}=E_{2}\left(0 ;(0, \theta) ;|1-z|^{2} ; N\right) .
$$

But a simple argument shows it is also true that

$$
\left.\frac{N}{2 \pi} E_{2}\left(0 ;(0, \theta) ; w_{2}(\theta)=1 ; N+1\right)\right|_{\text {given an eigenvalue at } \theta=0}=-\frac{d}{d \theta} E_{2}\left(0 ;(0, \theta) ; w_{2}(\theta)=1 ; N+1\right)
$$

where $N / 2 \pi$ is the density of eigenvalues at $\theta=0$, so we have the identity

$$
-\frac{2 \pi}{N} \frac{d}{d \theta} E_{2}\left(0 ;(0, \theta) ; w_{2}(\theta)=1 ; N+1\right)=E_{2}\left(0 ;(0, \theta) ;|1-z|^{2} ; N\right) .
$$

Putting

$$
E_{2}(0 ;(0, x) ; a) \equiv \lim _{N \rightarrow \infty} E_{2}\left(0 ;(0,2 \pi x / L) ;\left|1-e^{i \theta}\right|^{2 a} ; N\right)
$$

this implies

$$
-\frac{1}{\rho} \frac{d}{d x} E_{2}(0 ;(0, x) ; 0)=E_{2}(0 ;(0, x) ; 1)=\exp \left(-\int_{0}^{\pi \rho x} \frac{\tau_{1}(y)}{y} d y\right),
$$

where the second equality follows from (4.30) and Proposition 7.

We have noted the identity (4.42) in a recent Letter [7] presenting the exact Wigner surmise form for the spacing probability in the bulk of matrix ensembles with orthogonal or unitary symmetries. This is possible (in the case of unitary symmetry) because the spacing 
probability between consecutive eigenvalues, denoted $p_{2}(x)$ say, is related to $E_{2}(0 ;(0, x) ; 0)$ by

$$
p_{2}(x)=\frac{d^{2}}{d x^{2}} E_{2}(0 ;(0, x) ; 0)
$$

where the mean spacing in normalised so that $\rho=1$. Use of the formula (4.42) gives [7]

$$
p_{2}(x)=\frac{\tau_{1}(\pi x)}{x} \exp \left(-\int_{0}^{\pi x} \frac{\tau_{1}(y)}{y} d y\right)
$$

where $\tau_{1}$ satisfies the non-linear equation (4.32) with $a=1$ subject to the boundary condition

$$
\tau_{1}(x) \underset{x \rightarrow 0}{\sim} \frac{x^{3}}{3 \pi}
$$

Substituting for $E_{2}(0 ;(0, x) ; 0)$ on the left hand side of (4.42) according to (4.30) and the result of Proposition 8 shows that (4.42) implies an identity between transcendents, which in fact can be established directly from the theory in [3].

Proposition 9 The solutions $\tau_{0}(x)$ and $\tau_{1}(x)$ specified in Proposition 7 are related by

$$
\tau_{1}(x)=1+\tau_{0}(x)-x \frac{\tau_{0}^{\prime}(x)}{\tau_{0}(x)} .
$$

Proof - In Proposition 8 for the general Bäcklund transformations, we have in the case $a=0$

$$
\begin{aligned}
& -\tau_{0}=\frac{1}{4 u_{0}}\left[\frac{x u_{0}^{\prime}}{u_{0}-1}-u_{0}\right]^{2}+\frac{x^{2} u_{0}}{\left(u_{0}-1\right)^{2}}, \\
& -\tau_{0}^{\prime}=-\frac{x}{4 u_{0}\left(u_{0}-1\right)}\left[u_{0}^{\prime}-\frac{u_{0}\left(u_{0}-1\right)}{x}\right]^{2}-\frac{x u_{0}}{u_{0}-1} .
\end{aligned}
$$

so that if we form the quantity on the right-hand side of (4.46) then we find

$$
1+\tau_{0}(x)-x \frac{\tau_{0}^{\prime}(x)}{\tau_{0}(x)}=-\frac{1}{4 u_{0}}\left[\frac{x u_{0}^{\prime}}{u_{0}-1}-u_{0}\right]^{2}+u_{0}-\frac{x^{2} u_{0}}{\left(u_{0}-1\right)^{2}} .
$$

But now we note that the solution of the Painlevé transcendent for $a=0, u_{0}$, corresponds to $\alpha=1 / 2$, but is also identical to the one for $a=1, u_{1}$, because $\alpha$ is the same (taking the negative sign in (4.34)) and thus one can make the identification

$$
-\tau_{1}(x)=\frac{1}{4 u_{0}}\left[\frac{x u_{0}^{\prime}}{u_{0}-1}-u_{0}\right]^{2}-u_{0}+\frac{x^{2} u_{0}}{\left(u_{0}-1\right)^{2}},
$$

so that $(4.46)$ is established.

To understand that (4.44) is the simplest possible way of representing the spacing probability one only needs to observe that the identification of the solutions of two distinct second order second degree ODEs with one transcendent can only arise for $a=0,1$ in $\alpha=1 / 2(1-2 a)^{2}$. 


\section{The Double Interval}

We will now consider our second case, which is the probability of eigenvalues being excluded from the interval $(-\infty,-s) \cup(s, \infty)$, in a parallel manner to that of the previous case. We make the new conventions $a_{1}=-\infty, a_{2}=-s, a_{3}=s, a_{4}=\infty$, and $q_{3}, p_{3}=q$, $p$, with the symmetries $q_{2}=(-1)^{N} q, p_{2}=(-1)^{N-1} p$. We note the parity relation implies $v=0$.

Proposition 10 The coupled differential equations for the finite $N$ CyUE on the interval $(-\infty,-s) \cup(s, \infty)$ are

$$
\begin{aligned}
{\left[\ln E_{2}\right]^{\prime} } & =2 R \\
R(-s, s) & =(-1)^{N-1} \frac{q p}{s} \equiv(-1)^{N-1} R_{0}(s) \\
u^{\prime} & =-2 q^{2} \\
w^{\prime} & =-2 p^{2}, \\
\left(1+s^{2}\right) q^{\prime} & =-a s q+\left[\beta_{0}-u(2 a-1)\right] p+\frac{2\left(1+s^{2}\right)}{s} q^{2} p, \\
\left(1+s^{2}\right) p^{\prime} & =+a s p-\left[\gamma_{0}+w(2 a+1)\right] q-\frac{2\left(1+s^{2}\right)}{s} q p^{2}, \\
\left(1+s^{2}\right) R & =\left[\gamma_{0}+w(2 a+1)\right] q^{2}+\left[\beta_{0}-u(2 a-1)\right] p^{2}-2 a s q p+2 s\left(1+s^{2}\right) R_{0}^{2}, \\
{\left[\left(1+s^{2}\right) R\right]^{\prime} } & =-2 a q p-\frac{2\left(1+s^{2}\right)}{s^{2}} q^{2} p^{2} .
\end{aligned}
$$

Proof - These follow in an entirely parallel manner as for the single interval case.

Again such a system can be reduced to single second order differential equations for $R(s)$ or $R_{0}(s)$.

Proposition 11 The coupled set of ODEs given in Proposition 10 are equivalent to the following second order ODE for $\sigma(s)=\left(1+s^{2}\right) R(s)$,

$$
\begin{gathered}
\left\{\left(1-s^{2}\right) F-2 a s+\frac{s\left(1+s^{2}\right)}{F}\left[a^{2} s-2 s \sigma^{\prime}-\left(1+s^{2}\right) \sigma^{\prime \prime}\right]\right\}^{2} \\
-\left\{2\left(1+s^{2}\right) \sigma+2 a s^{2}(F-a s)-s(F-a s)^{2}\right\}^{2} \\
=-4 s^{2}(F-a s)^{2}\{N(N+2 a)-2 s \sigma-2 a s(F-a s)\}
\end{gathered}
$$

where $F \equiv \sqrt{a^{2} s^{2}-2\left(1+s^{2}\right) \sigma^{\prime}}$, with the boundary conditions

$$
\sigma(s) \underset{s \rightarrow \infty}{\sim} \frac{1}{2^{2 a+1} \Gamma(a+1 / 2) \Gamma(a+3 / 2)} \frac{\Gamma(N+2 a+1)}{\Gamma(N)} s^{-2 a} .
$$

Alternatively the coupled ODEs can be reduced to the following second order ODE for $R_{0}(s)$,

$$
\begin{gathered}
\left\{s\left(1+s^{2}\right)^{2} R_{0}^{\prime \prime}+2\left(1+s^{2}\right)\left(1+2 s^{2}\right) R_{0}^{\prime}+T\left[2 N(N+2 a)+1+s^{2}\right]-6 a T^{2}-4 T^{3}\right\}^{2} \\
=\left[a s-2\left(1-s^{2}\right) R_{0}\right]^{2}\left\{\left(1+s^{2}\right)^{2}\left(T^{\prime}\right)^{2}-4 T^{2}[T(T+2 a)-N(N+2 a)]\right\} .
\end{gathered}
$$


where $T(s) \equiv 2 s R_{0}=2 q p$, subject to the boundary conditions

$$
R_{0}(s) \underset{s \rightarrow \infty}{\sim} \frac{1}{2^{2 a+1} \Gamma(a+1 / 2) \Gamma(a+3 / 2)} \frac{\Gamma(N+2 a+1)}{\Gamma(N)} s^{-2 a-2} .
$$

Proof - In order to find our second order ODE we require an integral of the motion (not two as for the single interval case because $v=0$ ), and the construction of this parallels the earlier case. Firstly we note

$$
\left(1+s^{2}\right)(q p)^{\prime}+\left[\gamma_{0}+w(2 a+1)\right] q^{2}-\left[\beta_{0}-u(2 a-1)\right] p^{2}=0,
$$

using $(5.5,5.6)$ and

$$
\sigma-\left[\gamma_{0}+w(2 a+1)\right] q^{2}-\left[\beta_{0}-u(2 a-1)\right] p^{2}+2 a s q p-2 s^{-1}\left(1+s^{2}\right) q^{2} p^{2}=0,
$$

which is (5.7) rewritten. Now we employ a certain combination of these last two equations, namely (5.14) $+2 a$ times (5.13), which upon the use of (5.3,5.4) becomes

$$
\begin{aligned}
1 / 2 & \left\{\left[\gamma_{0}+w(2 a+1)\right]\left[\beta_{0}-u(2 a-1)\right]\right\}^{\prime} \\
+2 a\left(1+s^{2}\right)(q p)^{\prime}+4 a s q p & \\
& +\sigma-2 a s q p-2 s^{-1}\left(1+s^{2}\right) q^{2} p^{2}=0 .
\end{aligned}
$$

However the last two terms of this equation are just $s \sigma^{\prime}$ and thus the whole left-hand side is a perfect derivative. Utilising the boundary conditions as $s \rightarrow \infty$ we arrive at the integral of motion,

$$
\left[\gamma_{0}+w(2 a+1)\right]\left[\beta_{0}-u(2 a-1)\right]=\beta_{0} \gamma_{0}-2 s \sigma-4 a\left(1+s^{2}\right) q p .
$$

As our starting point in the reduction to an ODE in terms of $\sigma$ we consider the two equations, (5.13,5.14), rewritten in the following way

$$
\begin{aligned}
\left(1+s^{2}\right)(q p)^{\prime} & =\left[\beta_{0}-u(2 a-1)\right] p^{2}-\left[\gamma_{0}+w(2 a+1)\right] q^{2}, \\
\sigma+2 a s q p-2 s^{-1}\left(1+s^{2}\right) q^{2} p^{2} & =\left[\beta_{0}-u(2 a-1)\right] p^{2}+\left[\gamma_{0}+w(2 a+1)\right] q^{2} .
\end{aligned}
$$

Now we view (5.8) as a quadratic equation for $q p$, with

$$
q p=\frac{s}{2\left(1+s^{2}\right)}[F-a s],
$$

and use this relation and the definition of $F$ to express the left-hand sides of (5.17) solely in terms of $F$ and $\sigma$. Finally the difference of the squares of each are taken, and the integral of the motion (5.16) is used to eliminate the cross term. One is then left with the first of the second order ODEs (5.9).

For the second ODE we seek to first eliminate $\left[\gamma_{0}+w(2 a+1)\right] q^{2}$ and $\left[\beta_{0}-u(2 a-1)\right] p^{2}$ by employing (5.7) (the sum of these two terms), (5.13) (the difference of the two) and (5.16) (the cross product of the two). In this way we find the following quadratic equation for $\sigma$

$$
4\left\{\sigma+T\left[a s-\left(1-s^{2}\right) R_{0}\right]\right\}^{2}+4 T^{2}\{T(T+2 a)-N(N+2 a)\}-\left(1+s^{2}\right)^{2} T^{\prime 2}=0,
$$

and can then use this equation in conjunction with (5.8) to eliminate $\sigma$ entirely. The final result is the second ODE (5.11).

Remark - Knowledge of the above integral (5.16) allows us to express the auxiliary quantities $q, p, u, w$ in terms of the primary ones $\sigma, R_{0}$, and this will facilitate further understanding of the relationship of these to the transcendents and their scaling behaviour. 
Proposition 12 The auxiliary quantities $q, p$ are determined by quadrature from the two primary quantities $\sigma, R_{0}$ by the differential equations

$$
\begin{aligned}
& \left(1+s^{2}\right) \frac{q^{\prime}}{q}=\frac{\sigma}{2 s R_{0}}+\left(1+s^{2}\right) R_{0} \\
& \quad-\left\{\left[\frac{\sigma}{2 s R_{0}}+a s-\left(1+s^{2}\right) R_{0}\right]^{2}+2 s \sigma+4 a s\left(1+s^{2}\right) R_{0}-\beta_{0} \gamma_{0}\right\}^{1 / 2}, \\
& \left(1+s^{2}\right) \frac{p^{\prime}}{p}=-\frac{\sigma}{2 s R_{0}}-\left(1+s^{2}\right) R_{0} \\
& \quad-\left\{\left[\frac{\sigma}{2 s R_{0}}+a s-\left(1+s^{2}\right) R_{0}\right]^{2}+2 s \sigma+4 a s\left(1+s^{2}\right) R_{0}-\beta_{0} \gamma_{0}\right\}^{1 / 2},
\end{aligned}
$$

subject to the boundary conditions

$$
\begin{aligned}
& q(s) \underset{s \rightarrow \infty}{\sim} \frac{1}{\Gamma(a+1 / 2)}\left(\frac{2 a-1}{2 a+1}\right)^{1 / 4}\left\{\sqrt{N(N+2 a)} \frac{\Gamma(N+2 a)}{\Gamma(N+1)}\right\}^{1 / 2}(2 s)^{-a}, \\
& p(s) \underset{s \rightarrow \infty}{\sim} \frac{1}{\Gamma(a+3 / 2)}\left(\frac{2 a+1}{2 a-1}\right)^{1 / 4}\left\{\sqrt{N(N+2 a)} \frac{\Gamma(N+2 a+1)}{\Gamma(N)}\right\}^{1 / 2}(2 s)^{-a-1} .
\end{aligned}
$$

Proof - One starts by recasting (5.5) for $u$ in terms of $q$ and the primary quantities, namely

$$
\left[\beta_{0}-u(2 a-1)\right]=\frac{q}{s R_{0}}\left[\left(1+s^{2}\right) q^{\prime}+a s q-2\left(1+s^{2}\right) q R_{0}\right]
$$

and similarly for $\left[\gamma_{0}+w(2 a+1)\right]$ by using this result and the integral (5.16). One can then eliminate $u, w$ using these expressions in (5.17) thus relating $q, q^{\prime}$ solely in terms of $\sigma, R_{0}$ by a quadratic equation, and arriving at (5.20). The case for $p, p^{\prime}$ is virtually the same. The boundary conditions for $q, p$ follow from the asymptotic forms of $\phi(s), \psi(s)$ as $s \rightarrow \infty$ and their connection with Jacobi polynomials, (2.7,2.10). The sign of the radical is determined by a comparison of the boundary conditions for $q, p$ and $\sigma, R_{0}$.

The other auxiliary quantities $u, w$ can then be found from quadratures also, given $q, p$ using (5.3, 5.4).

\subsection{Reduction to Painlevé Transcendents}

The second degree second order equations of Proposition 11 can also be related to Painlevé transcendents.

Proposition 13 The variables $R_{0}(s), F(s)$, and $\sigma(s)$ are given in terms of the Painlevé-VI 
transcendent $\omega(x)$ by the formulae,

$$
\begin{aligned}
-2 i R_{0}= & \frac{\epsilon_{1} 2 x(x-1) \omega^{\prime}-(\omega-1)\left\{\epsilon_{1}(\omega+x)-2 a x\right\}}{2 \sqrt{x}(x-1) \omega}, \\
-i F= & \frac{\epsilon_{1} 2 x(1-x) \omega^{\prime}+\epsilon_{1}(\omega-1)(\omega+x)+2 a x}{2 \sqrt{x} \omega} \\
i \sigma=-\frac{\left\{2 x(x-1) \omega^{\prime}-(\omega-1)(\omega+x)\right\}^{2}}{8 \sqrt{x} \omega(\omega-1)(\omega-x)} & +\frac{\sqrt{x}(\omega-1)\left\{N(N+2 a) \omega+a^{2} x\right\}}{2 \omega(\omega-x)}
\end{aligned}
$$

where $\epsilon_{1}:= \pm 1$ and $s=i \sqrt{x}$, and with the parameters,

$$
\alpha=\frac{1}{8}, \quad \beta=-\frac{1}{8}(1 \mp 2 a)^{2}, \quad \gamma=0, \quad \delta=\frac{1}{2}\left(1-[N+a]^{2}\right) .
$$

Proof - Under the transformation $s \mapsto-i s$ and $R_{0} \mapsto-2 i R_{0}$ the equation (5.11) is identical in form to equation (5.14) in [14], and consequently the rest of the analysis there carries through with an adjustment of the transcendent parameters.

\subsection{Special Cases for low $N$}

In this part we present the analogous results for the first two finite- $N$ cases $N=1$ and $N=2$ by direct calculation of the probability $E_{2}(0 ; I)$.

Proposition 14 The probability (1.19) for $N=1$ and the associated functions are given by

$$
\begin{aligned}
E_{2}(0 ; I) & =\frac{2 \Gamma(a+1)}{\sqrt{\pi} \Gamma(a+1 / 2)} s_{2} F_{1}\left(a+1,1 / 2 ; 3 / 2 ;-s^{2}\right) \\
& =1-\frac{\Gamma(a+1)}{\sqrt{\pi} \Gamma(a+3 / 2)} s^{-2 a-1}{ }_{2} F_{1}\left(a+1, a+1 / 2 ; a+3 / 2 ;-s^{-2}\right) \\
\sigma(s) & =\frac{\left(1+s^{2}\right)^{-a}}{2 s_{2} F_{1}\left(a+1,1 / 2 ; 3 / 2 ;-s^{2}\right)} \\
F(s) & =a s+\frac{\left(1+s^{2}\right)^{-a}}{s_{2} F_{1}\left(a+1,1 / 2 ; 3 / 2 ;-s^{2}\right)}
\end{aligned}
$$


and for the corresponding probability with $N=2$ is

$$
\begin{aligned}
& E_{2}(0 ; I)=\frac{4 \Gamma(a+2) \Gamma(a+1)}{\pi \Gamma(a+1 / 2) \Gamma(a+3 / 2)} s_{2}^{2} F_{1}\left(a+2,1 / 2 ; 3 / 2 ;-s^{2}\right)\left[{ }_{2} F_{1}\left(a+1,1 / 2 ; 3 / 2 ;-s^{2}\right)-\left(1+s^{2}\right)^{-a-1}\right], \\
& =\left\{1-\frac{\Gamma(a+2)}{\sqrt{\pi} \Gamma(a+5 / 2)} s_{2}^{-2 a-3} F_{1}\left(a+2, a+3 / 2 ; a+5 / 2 ;-s^{-2}\right)\right\} \\
& \times\left\{1-\frac{2 \Gamma(a+2)}{\sqrt{\pi} \Gamma(a+3 / 2)} s_{2}^{-2 a-1} F_{1}\left(a+1, a+1 / 2 ; a+3 / 2 ;-s^{-2}\right)\right\}, \\
& \sigma(s)=\frac{\left(1+s^{2}\right)^{-a-1}}{2 s}\left\{\frac{2(a+1) s^{2}}{{ }_{2} F_{1}\left(a+1,1 / 2 ; 3 / 2 ;-s^{2}\right)-\left(1+s^{2}\right)^{-a-1}}+\frac{1}{{ }_{2} F_{1}\left(a+2,1 / 2 ; 3 / 2 ;-s^{2}\right)}\right\}, \\
& F(s)=a s+\frac{\left(1+s^{2}\right)^{-a-1}}{s}\left\{\frac{2(a+1) s^{2}}{{ }_{2} F_{1}\left(a+1,1 / 2 ; 3 / 2 ;-s^{2}\right)-\left(1+s^{2}\right)^{-a-1}}-\frac{1}{{ }_{2} F_{1}\left(a+2,1 / 2 ; 3 / 2 ;-s^{2}\right)}\right\},
\end{aligned}
$$

in terms of the Gauss hypergeometric function ${ }_{2} F_{1}(a, b ; c ; z)$.

Proof - We proceed in a parallel manner as in the proof of Proposition 6 and the relations given there.

Again one can show that these two specific cases are solutions of the second order differential equations (5.9,5.11) and satisfy the boundary conditions (5.10,5.12), using the contiguous and differentiation formulae for the Gauss hypergeometric function.

\subsection{The Thermodynamic Limit for the Circular Jacobi Ensemble}

In Section 4.3 we saw that the stereographic projection (4.28) maps, in the limit $L \rightarrow \infty$, the interval $(s, \infty)$ in the Cauchy ensemble to the interval $(0, x)$ in the scaled circular Jacobi ensemble. Similarly this mapping and limiting procedure maps the region $(\infty,-s) \cup(s, \infty)$ in the Cauchy ensemble to the interval $(-x, x)$ in the scaled circular Jacobi ensemble. Thus

$$
E_{2}\left(0 ;(-\infty,-s) \cup(s, \infty) ;\left(1+t^{2}\right)^{-N-a} ; N\right)=E_{2}\left(0 ;(-x, x) ;|1-z|^{2 a} ; N\right),
$$

and since with $\sigma(t)$ specified by Proposition 11

$$
E_{2}\left(0 ;(-\infty,-s) \cup(s, \infty) ;\left(1+t^{2}\right)^{-N-a} ; N\right)=\exp \left(-2 \int_{s}^{\infty} d t \frac{\sigma(t)}{1+t^{2}}\right)
$$

we have

$$
E_{2}\left(0 ;(-x, x) ;\left|1-e^{2 \pi i y / L}\right|^{2 a} ; N\right)=\exp \left(-\frac{2 \pi}{L} \int_{0}^{x} d y \sigma(\cot \pi y / L)\right)
$$

Furthermore, with

$$
\begin{aligned}
E_{2}(0 ;(-x, x) ; a) & =\lim _{\substack{N, L \rightarrow \infty \\
N / L=\rho}} E_{2}\left(0 ;(-x, x) ;\left|1-e^{2 \pi i y / L}\right|^{2 a} ; N\right) \\
\frac{1}{N} \sigma(\cot \pi y / L) & \mapsto \sigma(\pi \rho y)
\end{aligned}
$$


(5.31) gives

$$
E_{2}(0 ;(-x, x) ; a)=\exp \left(-2 \int_{0}^{\pi \rho x} d y \sigma(y)\right)
$$

It is of relevance to consider the scaled form of the Cauchy kernel under the stereographic projection (4.28). Using the relation between the Jacobi polynomial and the hypergeometric function ${ }_{2} F_{1}$, transformations and confluent forms of the latter, and the relationship of ${ }_{1} F_{1}$ to the Bessel function one can show [4]

$$
K_{N}\left(s_{1}, s_{2}\right) d s_{2} \rightarrow K\left(x_{1}, x_{2}\right) d x_{2}
$$

where

$$
K(x, y)=(\pi \rho x)^{1 / 2}(\pi \rho x)^{1 / 2} \frac{J_{a+1 / 2}(\pi \rho x) J_{a-1 / 2}(\pi \rho y)-J_{a+1 / 2}(\pi \rho y) J_{a-1 / 2}(\pi \rho x)}{2(x-y)} .
$$

This particular Bessel kernel was first identified in [11]. It has been used in [5] to provide a direct derivation of (5.34), and furthermore specify $\sigma(y)$ therein as the solution of a particular second order ODE. This latter result can be considered as a limiting case of (5.9). We summarise here the known results for this kernel, for use later on, and whilst we use the same symbols for primary and auxiliary quantities in this case and for the finite Cauchy problem the reader should not confuse them. We intend to display the interconnections between the scaled Cauchy quantities and the corresponding Bessel kernel ones in the following subsection.

Proposition 15 The gap probability and associated quantities for the Bessel kernel on the interval $(-x, x)$ with

$$
\begin{aligned}
& \phi(x)=(x / 2)^{1 / 2} J_{a+1 / 2}(x), \\
& \psi(x)=(x / 2)^{1 / 2} J_{a-1 / 2}(x),
\end{aligned}
$$

are defined by the following differential equations with $R \equiv R(x, x)$

$$
\begin{aligned}
u^{\prime} & =2 q^{2}, \\
w^{\prime} & =2 p^{2}, \\
x q^{\prime} & =x p+(-a+[u-w]) q, \\
x p^{\prime} & =-x q+(a-[u-w]) p, \\
x R & =x\left(q^{2}+p^{2}\right)+2(-a+[u-w]) q p+2(q p)^{2}, \\
(x R)^{\prime} & =q^{2}+p^{2} .
\end{aligned}
$$

along with the boundary conditions

$$
\begin{aligned}
& q(x) \underset{x \rightarrow 0}{\sim} \frac{1}{\Gamma(a+3 / 2)}(1 / 2 x)^{a+1}, \\
& p(x) \underset{x \rightarrow 0}{\sim} \frac{1}{\Gamma(a+1 / 2)}(1 / 2 x)^{a} .
\end{aligned}
$$


Proof - See reference [5], with the mapping $x \mapsto \pi \rho x$.

For future reference we note the following integral arising from (5.37,5.38,5.39,5.40),

$$
2 q p=w-u .
$$

Proposition 16 Consider the scaled function $\sigma(y)$ in (5.35), and write $\sigma_{1}(r) \equiv-2 x \sigma(x)$, $r \equiv 2 x$. Then we have that $\sigma_{1}$ satisfies the $O D E$

$$
\left(r \sigma_{1}^{\prime \prime}\right)^{2}+4\left[-a^{2}-\sigma_{1}+r \sigma_{1}^{\prime}\right]\left\{\left(\sigma_{1}^{\prime}\right)^{2}-\left[a-\sqrt{a^{2}+\sigma_{1}-r \sigma_{1}^{\prime}}\right]^{2}\right\}=0,
$$

subject to the boundary conditions

$$
\sigma_{1}(r) \underset{r \rightarrow 0}{\sim}-\frac{2}{\Gamma(a+1 / 2) \Gamma(a+3 / 2)}\left(\frac{1}{4} r\right)^{2 a+1} .
$$

Proof - The result comes from the leading order terms in the ODE (5.9) after the change of variables (4.28) and introducing the dependent function (5.33).

This is precisely the equation for $\sigma_{1}(2 x) \equiv-2 x R(x, x)$ that was derived from the coupled differential equations $(5.48-5.42)$ in [5]. We now want to indicate the precise relationships between the scaled Cauchy ensemble quantities $\sigma, R_{0}, q, p$ and the Bessel kernel quantities, which we shall distinguish by affixing the subscript $\infty$, namely the quantities $R_{\infty}, q_{\infty}, p_{\infty}$. From the arguments in the proposition above we see that

$$
\sigma(x)=R_{\infty}(x)
$$

after recognising that

$$
E_{2}(0 ;(-x, x) ; a)=\exp \left(-2 \int_{0}^{\pi \rho x} d y R_{\infty}(y)\right) .
$$

Having made this identification we can combine (5.41, 5.42) according to

$$
x \frac{d}{d x} \sigma_{1}-\sigma_{1}=-4 a\left(q_{\infty} p_{\infty}\right)-4\left(q_{\infty} p_{\infty}\right)^{2} .
$$

Then by performing the scaling limit on (5.8) and comparing with the above equation we find a simple scaling for $R_{0}$

$$
N R_{0}(s)=r(\pi \rho x)
$$

along with the identification in the thermodynamic limit

$$
r(x)=x q(x) p(x)=x q_{\infty}(x) p_{\infty}(x) .
$$

Here we define the scaled auxiliary variables by $q(s) \mapsto q(\pi \rho x)$ and $p(s) \mapsto p(\pi \rho x)$. Utilising these two simple scalings we have the following result.

Proposition 17 The scaled limits of the auxiliary quantities for the Cauchy ensemble on the double interval $q(x), p(x)$ are related to the auxiliary variables for the Bessel kernel $q_{\infty}(x), p_{\infty}(x)$ by

$$
\begin{aligned}
& q(x)=\frac{1}{\Gamma(a+1 / 2)}\left(\frac{2 a-1}{2 a+1}\right)^{1 / 4}(1 / 2 x)^{a} \exp \left(-\int_{0}^{x} d y \frac{q_{\infty}(y)}{p_{\infty}(y)}\right), \\
& p(x)=\frac{1}{\Gamma(a+3 / 2)}\left(\frac{2 a+1}{2 a-1}\right)^{1 / 4} \frac{(1 / 2)^{a+1}}{x^{a}} \exp \left(+\int_{0}^{x} d y \frac{p_{\infty}(y)}{q_{\infty}(y)}\right) .
\end{aligned}
$$


Proof - Utilising the scaling forms (5.33,5.50) in the expressions for the logarithmic derivatives of $q$ in $(5.20)$ we find

$$
\frac{q^{\prime}}{q}-a x^{-1}=-\frac{q_{\infty}}{p_{\infty}} \quad \text { or } \quad-\frac{p_{\infty}}{q_{\infty}}
$$

The correct choice can be made and the integration constant found by imposing both the boundary conditions (5.21,5.43). The situation for $p$ is entirely analogous.

\subsection{Reduction of scaled Limit to Painlevé Transcendents}

In this part we show how to reduce the auxiliary variables $q, p$ (we now drop the subscript $\infty)$ for the Bessel kernel directly to Painlevé transcendents.

Proposition 18 The variable $p(x)$ for the Bessel function kernel with parameter $a$ on the interval $(-x, x)$ is related to the Painlevé- $V$ transcendent $y(x)$ by

$$
p=-\sqrt{\frac{x}{2}} \frac{1+y}{1-y}
$$

whose parameter values are

$$
\alpha=\frac{1}{32}(1-2 a)^{2}, \quad \beta=-\frac{1}{32}(1-2 a)^{2}, \quad \gamma=0, \quad \delta=-2 .
$$

In terms of this transcendent $q(x), \sigma_{1}(2 x)$ are

$$
\begin{aligned}
q & =\sqrt{\frac{2}{x}} \frac{1}{4 y}\left[-x \dot{y}+\frac{1}{4}(2 a-1)\left(1-y^{2}\right)\right], \\
\sigma_{1} & =\frac{1}{y}\left[\frac{x \dot{y}}{1-y}+1 / 4(1+y)\right]^{2}-1 / 4 a^{2} \frac{(1+y)^{2}}{y}-x^{2}\left(\frac{1+y}{1-y}\right)^{2} .
\end{aligned}
$$

Proof - We eliminate all variables other than $q$ and $p$ from equations (13)-(17) in [5] using (5.44), and employ this with the coupled set (5.39,5.40). Elimination of $q$ using

$$
q=\frac{x p^{\prime}-a p}{2 p^{2}-x}
$$

leads to the ODE for $p$,

$$
p^{\prime \prime}=\frac{2 p}{2 p^{2}-x}\left(p^{\prime}\right)^{2}-\frac{2 p^{2}}{x\left(2 p^{2}-x\right)} p^{\prime}+\frac{p}{x}\left(2 p^{2}-x\right)+a(1-a) \frac{p}{x\left(2 p^{2}-x\right)} .
$$

Then applying the above transformation reduces this to a Painlevé-V. 


\section{Conclusions}

The circular Jacobi ensemble describes a degeneracy of order $a$ in the spectrum of unitary random matrices. We have found the probability that the one-sided interval $(0, x)$ to the right of the singularity is eigenvalue free, and similarly for the symmetrical interval $(-x, x)$ about the singularity. The former probability has been expressed in terms of a Painlevé-VI transcendent, which in the scaled limit reduces to a Painlevé-V transcendent. These calculations were performed by first mapping the circular Jacobi ensemble, via a stereographic projection, to the Cauchy ensemble of random Hermitian matrices. This latter ensemble is classical, having the same essential properties as the more familiar Hermite, Laguerre and Jacobi classical ensembles. As the probability of a gap free interval about an endpoint of these latter three ensembles are known in terms of Painlevé type transcendents from earlier studies [13, 8], as is the probability for two intervals at the ends of the support in the Hermite and symmetric Jacobi cases [14], by studying the Cauchy ensemble we are completing a classification program of determining the Painlevé transcendents associated with such probabilities in the classical ensembles.

The Cauchy ensemble is closely related to the symmetric Jacobi ensemble. Previous studies of the probabilities of gap free regions in this latter ensemble [13, 8, 14] have encountered third order differential equations, which through various means have been integrated to second order equations. In contrast in this study we are able to find an extra integral of the coupled differential equations, unknown from previous studies, which provides a direct path to the second order differential equation characterising the gap probability. We consider that the method of constructing this new integral will apply more generally than just to the ensemble treated here and will have important implications for the application of the Tracy and Widom formalism to other problems.

The case $a=1$ of the scaled circular Jacobi ensemble has particular relevance to the scaled CUE of random unitary matrices, or equivalently to the scaled GUE of random Hermitian matrices. Thus it corresponds to fixing an eigenvalue at the origin in these latter two scaled ensembles. The probability of no eigenvalues in the one-sided interval $(0, x)$ of the scaled circular Jacobi ensemble is then proportional to the derivative of the probability that there are no eigenvalues in the interval $(0, x)$ of the scaled CUE or GUE. This is turn allows us to derive the exact Wigner surmise type form (4.44) for the spacing probability between consecutive eigenvalues in the bulk of matrix ensembles with unitary symmetry.

\section{References}

[1] M. Adler, P. J. Forrester, T. Nagao, And P. van Moerbeke, Classical skew orthogonal polynomials and random matrices, (1999).

[2] R. Askey, Beta integrals and the associated orthogonal polynomials, in Lecture Notes in Mathematics, vol. 1395, Springer Verlag, Berlin - New York, 1989, pp. 84-121.

[3] C. M. Cosgrove And G. Scoufis, Painlevé classification of a class of differential equations of the second order and second degree, Stud. Appl. Math., 88 (1993), pp. 2587.

[4] P. J. Forrester, Random Matrices and Log Gases. Book in preparation. 
[5] P. J. Forrester And A. M. Odlyzko, Gaussian unitary ensemble eigenvalues and Riemann $\zeta$ function zeros: A nonlinear equation for a new statistic, Phys. Rev. E, 54 (1996), pp. 4493-4495.

[6] P. J. Forrester And E. M. RAIns, Inter-relationships between orthogonal, unitary and symplectic matrix ensembles. solv-int/9907008.

[7] P. J. Forrester And N. S. WitTe, Exact Wigner surmise type evaluation of the spacing distribution in the bulk of the scaled random matrix ensembles, Lett. Math. Phys., (2000).

[8] L. Haine And J.-P. Semengue, The Jacobi polynomial ensemble and the Painlevé VI equation, J. Math. Phys., 40 (1999), pp. 2117-2134.

[9] A. R. Its, A. G. Izergin, V. E. Korepin, and N. A. Slavnov, Differential equations for quantum correlation functions, Int. J. Mod. Phys. B, 4 (1990), pp. 1003-1037.

[10] M. Jimbo, T. Miwa, Y. Mori, and M. Sato, Density matrix of an impenetrable Bose gas and the fifth Painlevé transcendent, Physica D, 1 (1980), pp. 80-158.

[11] T. Nagao And K. Slevin, Nonuniversal correlations for random matrix ensembles, J. Math. Phys., 34 (1993), pp. 2075-2085.

[12] V. Romanovski, Calcul des probabilities - sur queques classes nouvelles de polynomes orthogonaux, Comptes Rendus Seances Acad. Sci., 188 (1929), pp. 1023-1025.

[13] C. A. Tracy and H. Widom, Fredholm determinants, differential equations and matrix models, Commun. Math. Phys., 163 (1994), pp. 33-72.

[14] N. S. Witte, P. J. Forrester, And C. M. Cosgrove, Gap probabilities for edge intervals in finite Gaussian and Jacobi unitary matrix ensembles, Nonl., (2000).

\section{Acknowledgements}

Both authors acknowledge the support of an Australian Research Council Grant whilst this work was performed, and NSW thanks Andrew Hone and Christopher Cosgrove for discussions. 